\title{
Tratamiento combinado de los queloides mediante cirugía y braquiterapia
}

\section{Treatment of queloids with surgical resection and brachytherapy}

\author{
Bisbal J .*, Guix B. **, Coronel R.*
}

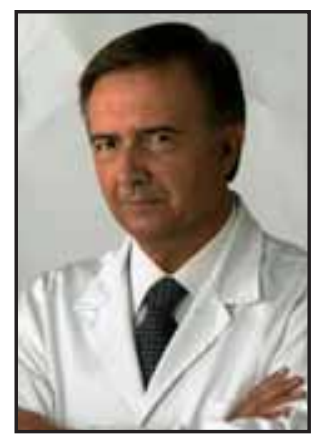

Bisbal, J.

\section{Resumen}

Presentamos nuestra experiencia en el tratamiento de los queloides mediante resección quirúrgica y radioterapia inmediata posterior.

La radioterapia transforma el queloide en un tejido hipocelular, hipovascular e hipóxico, impidiendo la excesiva migración de fibroblastos. Si se administra la dosis adecuada de radiación, se consigue un equilibrio entre la formación de cicatriz y la proliferación excesiva de tejido, evitando la formación de queloide, sin impedir la cicatrización normal.

Nuestro protocolo se divide en dos fases: la primera consiste en la exéresis del queloide mediante una incisión que sigue el trayecto del mismo, con un cuidadoso cierre en dos planos siendo siempre el superficial una sutura intradérmica. La segunda fase será la radioterapia, con dos modalidades posibles de tratamiento: 1- Braquiterapia (tratamiento a corta distancia), requiere la colocación de un catéter bajo la sutura, a través del cual se introduce una fuente radiactiva, normalmente Ir192. Se emplea especialmente en heridas longitudinales. 2- Radioterapia externa con electrones de baja energía. Se emplea en heridas complejas o extensas.

Generalmente, dosis de $20 \mathrm{~Gy}$. en 4 fracciones de 500 cGy, ofrecen excelentes resultados con mínimas secuelas o efectos secundarios. El volumen blanco debe incluir la herida quirúrgica más un margen de $4 \mathrm{~mm}$. alrededor de ella.

Con este procedimiento, hemos observado unos excelentes resultados, con un índice de recidivas inferior al $4 \%$, y una mejora en los síntomas clínicos en el $100 \%$ de los casos. Como efectos secundarios observamos telangiectasias $(15,4 \%)$ o cambios en la pigmentación cutánea $(5,9 \%)$.

\section{Palabras clave Cicatriz, Queloide, Braquiterapia.}

We present our experience in treatment of keloid scars with surgical resection and immediately postoperative radiotherapy.

Radiotherapy changes keloid into an hypocellular, hypovascular and hypotoxic tissue, avoiding the excessive migration of fibroblasts. If the suitable dose of radiation is delivered, balance between the formation of the scar and the excessive proliferation of the tissue is achieved avoiding the formation of the keloid but keeping normal scarring.

Our protocol is divided in two phases: the first one is the surgical treatment. The exeresis of the keloid is carried out through an incision that follows its own line and a careful closure in two planes; superficial one is always an intradermic suture. The second part will be radiotherapy, with two possible treatments: 1) Brachitherapy (treatment at a short distance), placing a catheter under the suture, through which a radioactive source is introduced, usually Ir192. It is used, specially in longitudinal wounds. 2) External radiotherapy with electrons of low energy. It is used in complex or large wounds.

A dose of $20 \mathrm{~Gy}$., in 4 fractions of $500 \mathrm{cGy}$, usually offers excellent results with minimum sequelae or side effects. The area to be irradiated should include the surgical wound, and a margin of $4 \mathrm{~mm}$. around it.

With this procedure, we have observed excellent results with a recidive rate lower tan $4 \%$ and an improvement in the clinical symptoms in $100 \%$ of cases. As a side effect, we have observed telangiectasies $(15,4 \%)$ or changes in the cutaneous pigmentation $(5,9 \%)$.
Key words $\quad$ Scars, Keloids, Brachiteraphy.

Numeral Gode $\quad 153-1532-140$ 


\section{Introducción}

Las cicatrices hipertróficas o en el peor de los casos queloideas, son uno de los mayores enemigos del cirujano plástico y su aspecto puede condicionar un buen o un mal resultado en una cirugía.

Desde la presentación de los trabajos de Tredget en 1997, (1) se han producido importantes avances en el conocimiento de la fisiopatología del proceso de cicatrización de las heridas, una herida es una condición fisiopatológica que parece estar regulada por diversos factores de crecimiento celular y por un equilibrio entre diferentes enzimas degradadores de la matriz tisular y sus inhibidores. Durante el proceso de cicatrización normal, tiene lugar una respuesta celular altamente coordinada tendente a restaurar la función y anatomía del tejido dañado. En la cicatrización de las heridas de tejidos blandos, los procesos fundamentales que suceden son: angiogénesis, formación de la matriz de colágeno y epitelización. Una vez que la herida está totalmente cerrada, debe inducirse la apoptosis celular (muerte celular inducida), de forma que se restablezca un equilibrio entre la formación de tejido y la aparición de una cicatriz de aspecto normal. El factor que induce la apoptosis celular es la cascada de citoquinas, que inhibe los factores de crecimiento celular y plasmático. Cuando una de las citoquinas está alterada, la inhibición de los factores de crecimiento es incompleta y la formación de tejido cicatricial continua, conduciendo a un exceso de síntesis de colágeno, fibronectina y proteoglicanos. La degradación deficiente de la matriz intersticial y el remodelado cicatricial inadecuado, dan lugar a un aspecto rojo, hipersensible, abultado y duro que es característico de los queloides. La incidencia de presentación de queloides en todas las heridas es del 5 al $15 \%$.

Podemos definir el queloide, como un tumor benigno cutáneo, secundario a una agresión tisular, caracterizado por una proliferación anormal de tejido conjuntivo, que puede extenderse mucho más allá del área de la herida y que no regresa espontáneamente. Desde el punto de vista histológico, veremos bandas de colágeno gruesas, hialinizadas, dispuestas en haces, así como gran cantidad de fibroblastos que tienden a orientarse en el mismo sentido que los haces de colágeno. Clínicamente se caracteriza por ser un tumor duro, de epidermis fina y lisa, de color rojo o púrpura, que puede tener zonas de ulceración focalizadas. Causa problemas de quemazón, prurito, dolor y en ciertos casos puede conducir a una importante limitación e incapacidad funcional.

Es importante establecer el diagnóstico diferencial con la cicatriz hipertrófica. Esta nunca se extiende fuera del área lesionada y tiende a la regresión espon- tánea. Histológicamente también apreciamos diferencias: presentan haces de colágeno no hialinizados y bastantes fibroblastos con vasos perpendiculares a la superficie dérmica.

Aunque se han descrito queloides de aparición espontánea o como consecuencia de traumas mínimos como pequeñas infecciones o arañazos en la piel, fundamentalmente se presentan de forma secundaria a lesiones cutáneas por traumatismo, infección o cirugía.

Existen factores predisponentes para la aparición del queloide tales como: herencia, localización en determinadas regiones del cuerpo, quemaduras y raza negra. Hasta la fecha, no se ha detectado ningún gen que pudiese ser responsable de esta mayor predisposición personal

Suelen aparecer a las 3 ó 4 semanas del traumatismo y se desarrollan hasta los 2 ó 3 meses, no remitiendo espontáneamente.

Se han propuesto muchos tratamientos con altos índices de recidiva, siendo el peor la exéresis quirúrgica simple, ya que recidiva en más del $90 \%$ de los casos y los empeora en más del $30 \%(2,3)$. Otros procedimientos como infiltraciones intralesionales con corticoides (4), presoterapia, crioterapia (5), láser (6), láminas de silicona (7-9) y diversos procedimientos combinados (10), han tenido unos índices de remisión muy escasos y un nivel de recidivas muy alto.

Existe una amplia experiencia sobre el uso de la radioterapia en los queloides y muchos autores han descrito procedimientos (11-15) que con el tiempo se han ido mejorando, especialmente por la evolución de los medios técnicos disponibles en la actualidad.

La radioterapia transforma el queloide en un tejido hipocelular, hipovascular e hipóxico, impidiendo la excesiva migración de fibroblastos y la formación del queloide. Si se administra la dosis adecuada de radiación, se consigue un equilibrio entre la formación de cicatriz y el excesivo crecimiento celular, evitando así la formación del queloide sin retrasar la cicatrización normal. Los estudios realizados por Chang en 1998 (16), sobre el factor de crecimiento básico de fibroblastos (bFGF), demuestran que la radiación acelera la apoptosis celular reduciendo la vascularización y la producción de fibrosis.

En este estudio presentamos nuestra experiencia en el tratamiento de los queloides mediante resección quirúrgica y radioterapia posterior inmediata, con el que como veremos, hemos conseguido una importante mejoría en los resultados

\section{Material y método}

El presente trabajo está basado en una recopilación de pacientes tratados por padecer queloides, mediante 


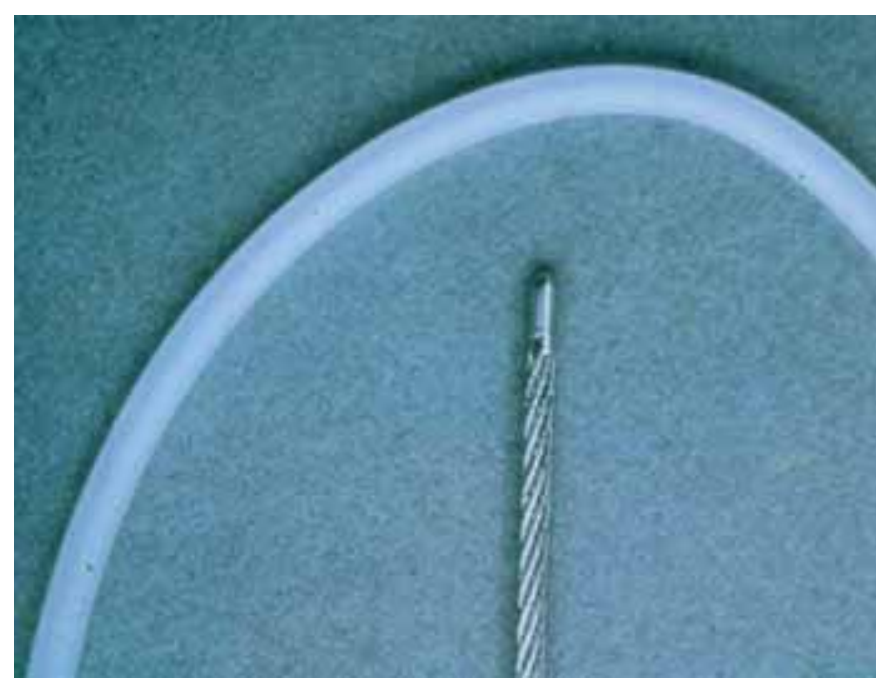

Fig. 1. Catéter para colocación subcutánea y fuente radiactiva Ir192.

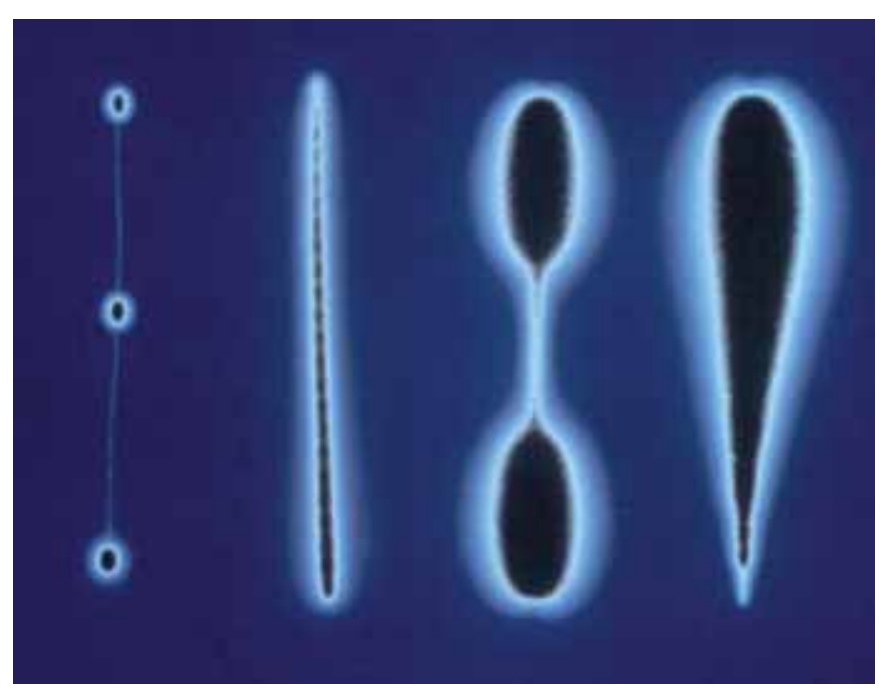

Fig. 2. Ejemplos de distribución de dosis en la Braquiterapia.

terapia combinada de exéresis quirúrgica y radioterapia inmediata posterior. Fue realizado durante el periodo comprendido entre los años 1999 y 2008, con un total de 48 pacientes tratados, 26 de sexo masculino y 22 de sexo femenino, con edades comprendidas entre los 24 y los 52 años.

Las áreas corporales tratadas fueron especialmente, la región mamaria, la región retroauricular, las orejas, los hombros, la región esternal, la cara, la espalda y el abdomen, con la siguiente distribución: mamas 5 casos, hombros 9, región esternal 11, cara 4, espalda 7 , orejas 9 , región retroauricular 2 y abdomen 1 .

Podemos dividir nuestro protocolo de actuación frente a las cicatrices queloideas en dos fases principales. La primera, consiste en el tratamiento quirúrgico del queloide y la segunda en la aplicación inmediata de radioterapia.

Primera fase: la exéresis del queloide debe realizarse bajo anestesia local o local con sedación según el tamaño de las cicatrices, con una incisión que siga el trayecto del mismo. Es conveniente efectuar una extirpación completa, o casi completa, del tejido queloideo, lesionando lo menos posible los tejidos sanos vecinos. En queloides muy grandes (mayores de $3 \mathrm{a} 4 \mathrm{~cm}$.) se deben aproximar los márgenes quirúrgicos mediante sutura subcutánea para evitar excesiva tensión en la piel. La hemostasia debe ser muy cuidadosa. El cierre externo debe realizarse mediante sutura continua intradérmica para limitar al máximo el daño sobre la piel. Para reducir aún más la tensión cutánea, abrimos la herida con bandas de steri-strip. En algunas zonas como las orejas, se requiere no solo la extirpación, sino la reconstrucción inmediata del defecto, ya que el queloide suele invadir y destruir tejidos sanos y con ello, provocar deformaciones.

Segunda fase: es el tratamiento con radioterapia, del que existen dos modalidades posibles:

1- La braquiterapia, que significa tratamiento a distancia corta, preferiblemente HDR (Alta tasa de dosis).

2- La radioterapia externa con electrones de baja energía.

Braquiterapia: requiere la colocación de un catéter en el mismo lecho quirúrgico de extirpación del queloide (Fig. 1), a través del cual se introduce una fuente radiactiva, normalmente Ir192. La fuente tiene unos $3 \mathrm{~mm}$ de longitud y 1,2 mm de diámetro y está soldada al final de un cable metálico. Su movimiento por el interior del catéter está controlado por ordenador; en este caso se trata de un Microselectrón que contiene la fuente radiactiva y los motores que la mueven por el interior del catéter. Variando el tiempo en que la fuente radiactiva permanece parada en unos puntos determinados, conseguimos distintas distribuciones de la dosis según requerimientos. Si por ejemplo, la hacemos estar unos segundos en una misma posición y desplazarse rápidamente a otro punto, obtendremos una distribución de dosis "puntiforme". Si por el contrario la hacemos viajar a puntos muy próximos entre sí, conseguimos una distribución de dosis "filiforme". (Fig. 2). De esta manera, mediante la optimización de la distribución de dosis con ordenador, podemos conseguir un tratamiento individualizado, con una distribución de dosis ideal y con una mínima irradiación de los tejidos sanos.

Empleamos dosis de radiación de 10 a 20 Gy en 1 a 5 fracciones. Dosis mayores producen efectos secundarios y retraso en la cicatrización, así como peores resultados estéticos a largo plazo con la aparición de atrofia cutánea y de telangiectasias en la zona tratada. Generalmente, la dosis de 20 Gy en 4 fracciones de 500 cGy (centigray) 2 veces al día, en 2 días, calculados a $4 \mathrm{~mm}$ bajo la superficie cutánea, ofrece excelentes resultados con mínimas secuelas o efectos secundarios. El volumen blanco, debe incluir la herida quirúrgica más un margen de $4 \mathrm{~mm}$ alrededor de 


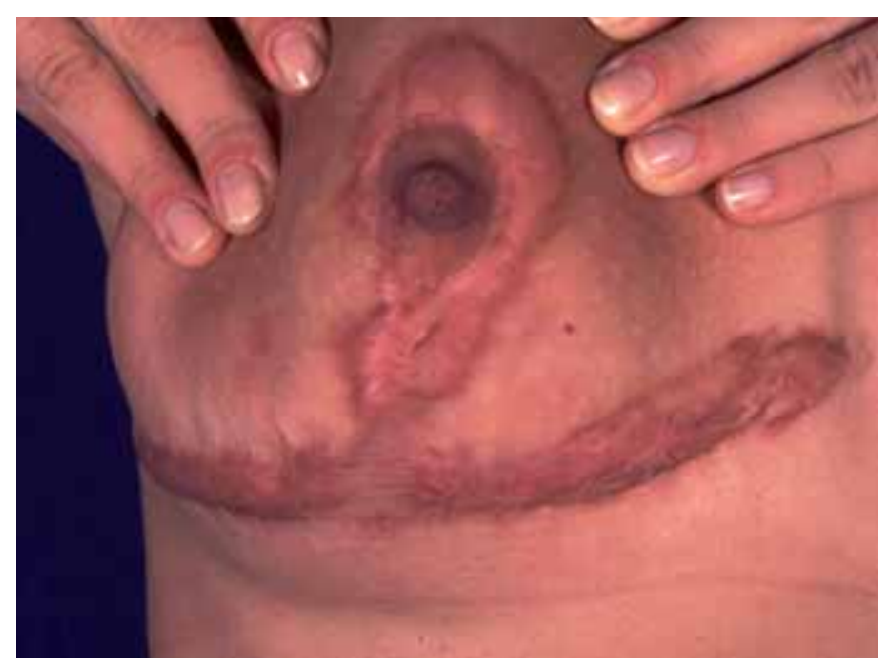

Fig. 3. Queloide en cicatrices de reducción mamaria. Puede verse su invasión a tejidos sanos.

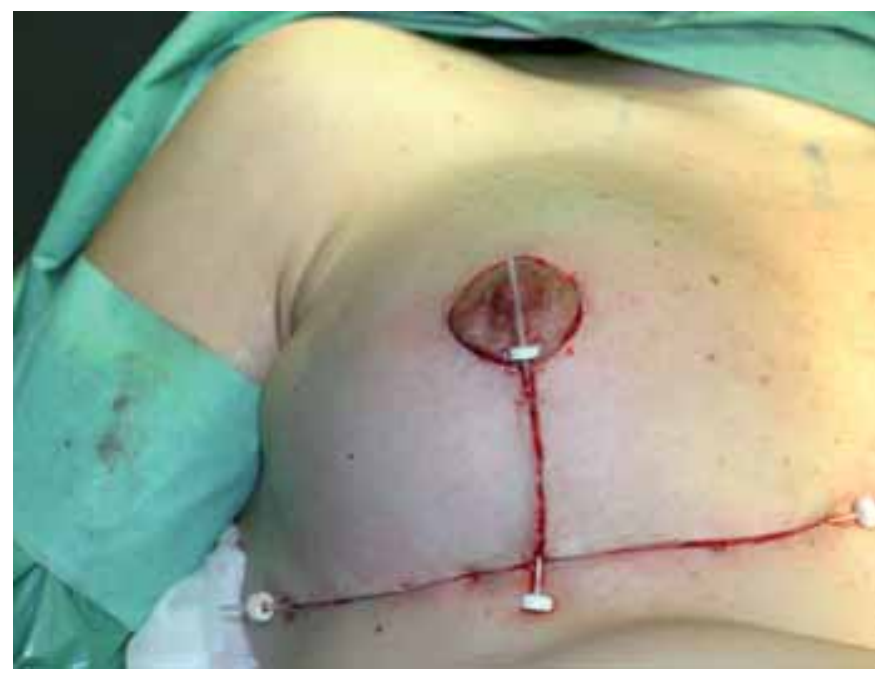

Fig. 4. Extirpación quirúrgica del queloide y colocación de un catéter bajo la sutura. (Braquiterapia).

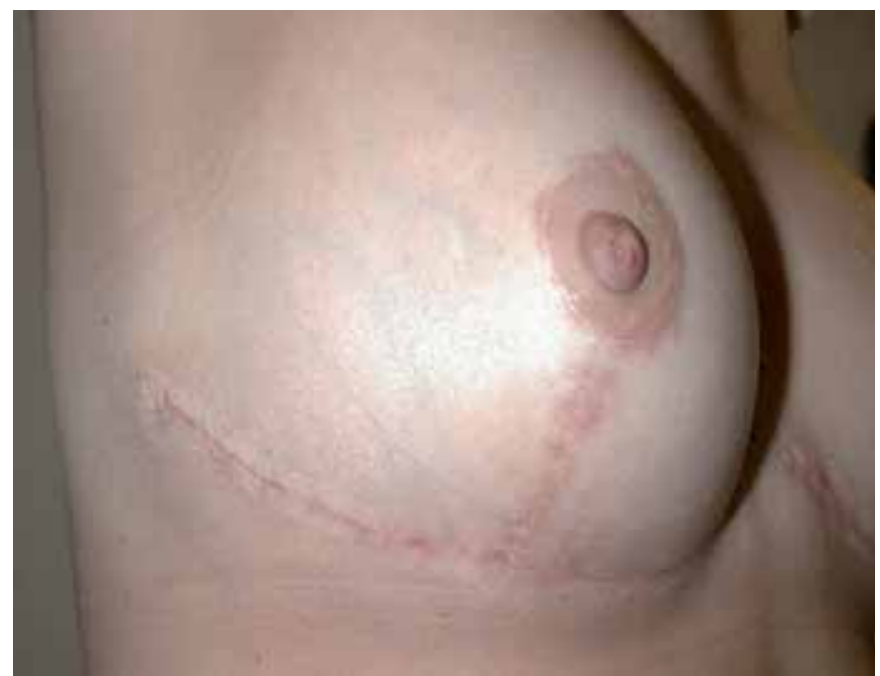

Fig. 5. Resultado a los 6 meses del tratamiento.

ella. Es importante empezar el tratamiento lo antes posible. Generalmente la empleamos para el tratamiento de queloides con un trayecto lineal y en superficies planas o curvas.
Entre las principales ventajas de esta técnica destacamos que la radiación empleada tiene muy corto alcance, por tanto irradia muy poca cantidad de tejido sano, con lo que la radioprotección del paciente es máxima. El principal inconveniente es la necesidad de llevar el catéter en la zona de la herida, por lo que no podrá usarse en casos muy extensos o complejos.

Radioterapia externa: es el tratamiento de la herida dejada por la extirpación de un queloide mediante radiaciones, estando situada la fuente emisora en el exterior del paciente. En este caso utilizamos radiaciones con poca capacidad de penetración, preferentemente electrones de baja energía, ya que el queloide es un proceso superficial y se deben minimizar los posibles riesgos secundarios: electrones de $9 \mathrm{MeV}$., calculando la dosis a $4 \mathrm{~mm}$ de profundidad de la piel, administrando igualmente 20 Gy en 4 sesiones de 500 cGy en 2 días (a razón de 2 sesiones al día), El campo de irradiación abarca la cicatriz quirúrgica externa y un margen lateral de $5 \mathrm{~mm}$ a cada lado.

La utilizamos en aquellos queloides en los que tras su extirpación no es posible colocar un catéter bajo la herida, bien por tratarse de una zona múltiple y reducida o bien por su localización, como por ejemplo en las orejas.

Su mayor ventaja está en que su dosimetría es más simple que para la braquiterapia. Entre los inconvenientes, el que irradia mayor cantidad de tejido sano, por lo tanto la radioprotección es menor que con la Braquiterapia.

Entre los 48 casos del estudio, en 14 empleamos braquiterapia y en 34 radioterapia externa.

\section{Resultados}

Comprobamos que en todos los casos de nuestro estudio sobre cicatrices queloideas tratadas con la técnica combinada de exéresis quirúrgica y radioterapia inmediata posterior, obtuvimos una mejoría muy positiva y aunque evidentemente la cicatriz persiste, su aspecto, tamaño, dureza, color, tendencia expansiva y especialmente la sintomatología clínica acompañante, mejoraron sustancialmente. El seguimiento de estos pacientes se ha realizado entre 6 meses y 3 años, y se ha hecho mediante controles fotográficos y medición de las cicatrices, aunque en el caso de los queloides a diferencia de las cicatrices hipertróficas, el cambio clínico tanto objetivo como subjetivo por parte del paciente es tan evidente que es difícil aplicarle escalas predeterminadas, sobre todo en regiones como las orejas, en las que el queloide se manifiesta como un tumor, a veces de grandes dimensiones y cuyo resultado positivo tras el tratamiento es que no vuelva a salir. En los queloides 

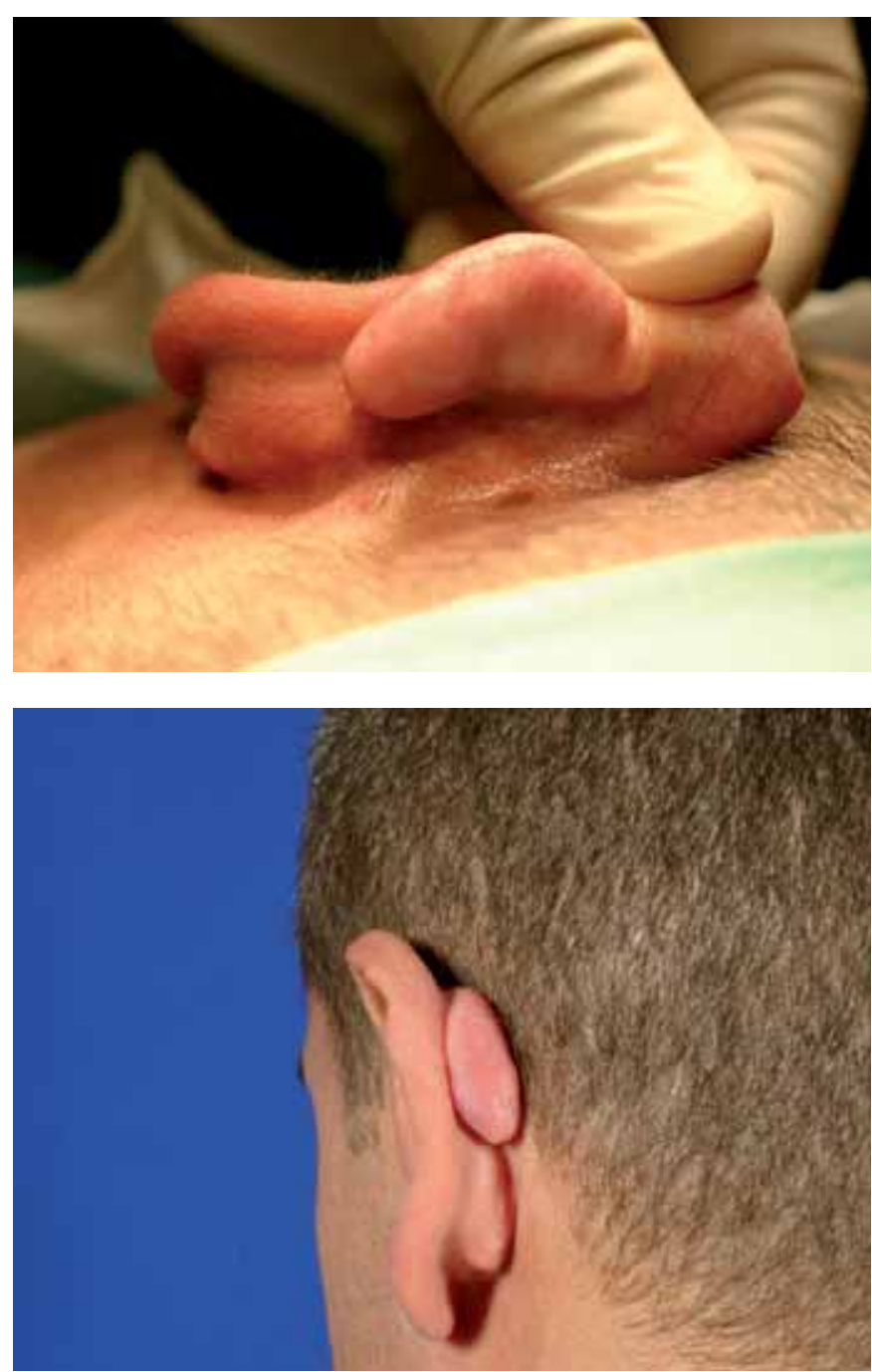

Fig. 6 y 7. Queloide retroauricular tras intervención de otoplastia.

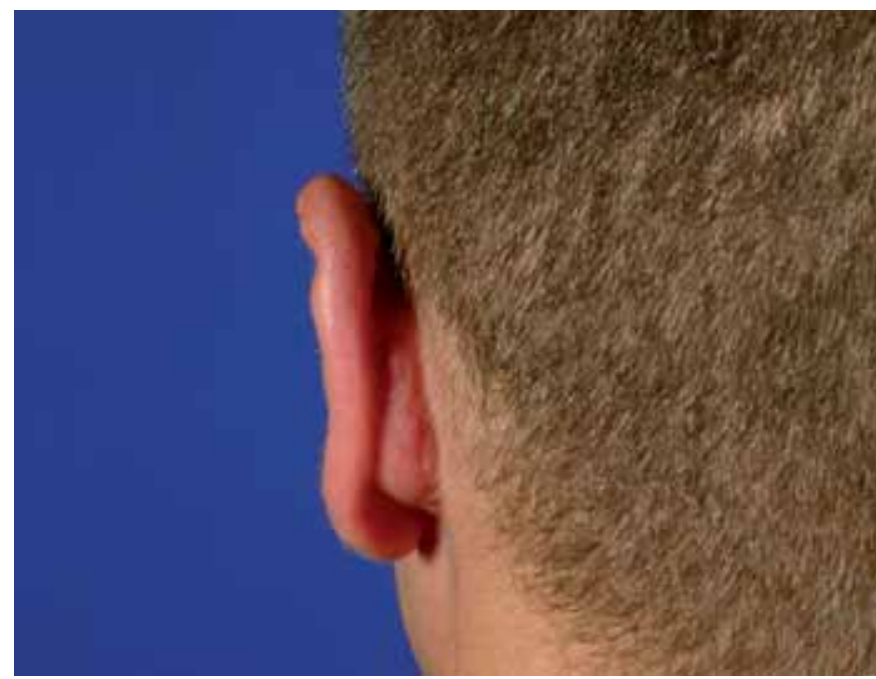

Fig. 8. Resultado al año del tratamiento. (Radioterapia externa).

de extensión plana puede aplicarse para su seguimiento la escala de Vancouver. Las recidivas totales, entendiéndose como tales la aparición de un queloide de tamaño similar al extirpado, con las dosis antes mencionadas, han estado por debajo del $4 \%$ de los casos, siendo la espalda la región que comprende la mayoría de estas recidivas. No hemos podido esta-

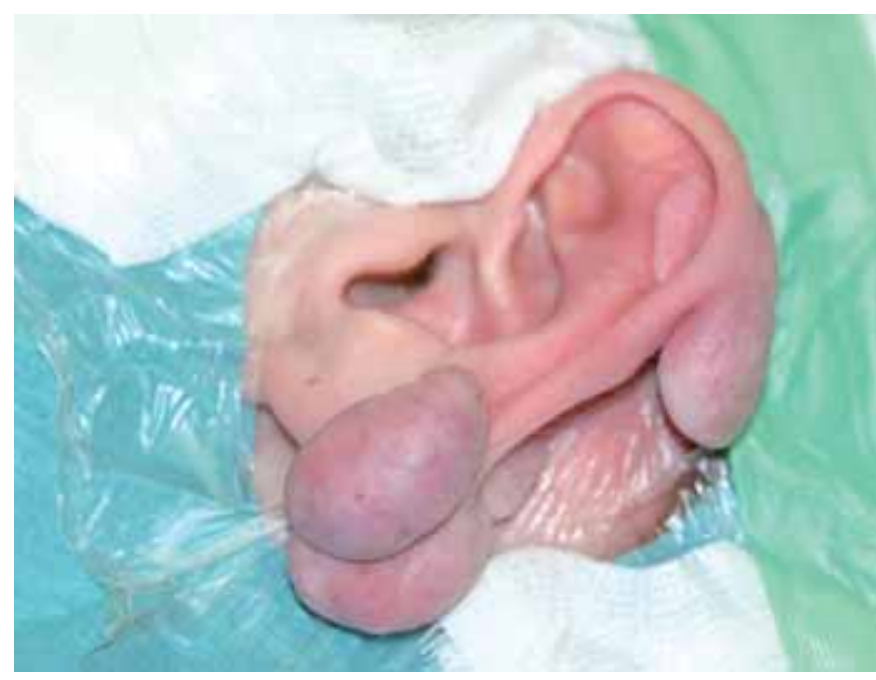

Fig. 9. Queloides múltiples en oreja producidos por peercing.

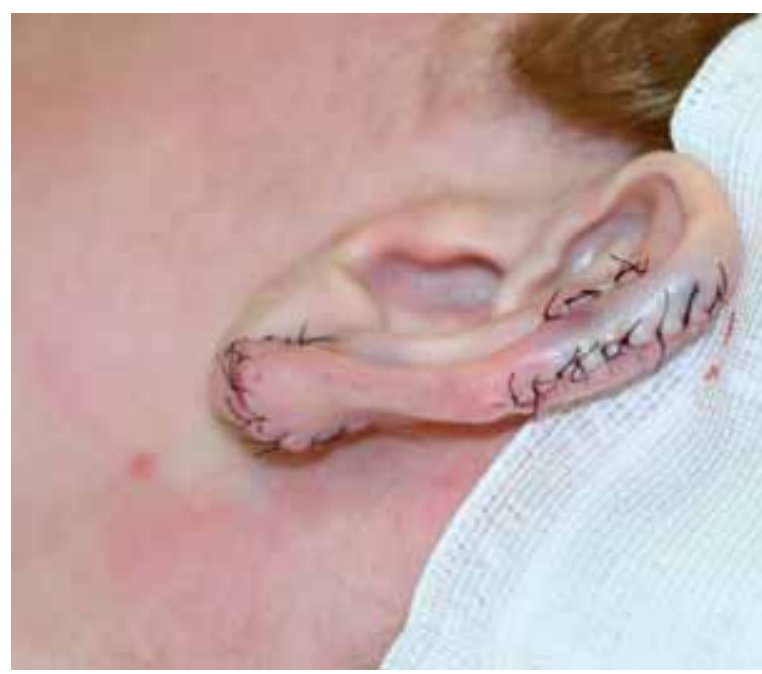

Fig. 10. Resultado tras exéresis y reconstrucción.

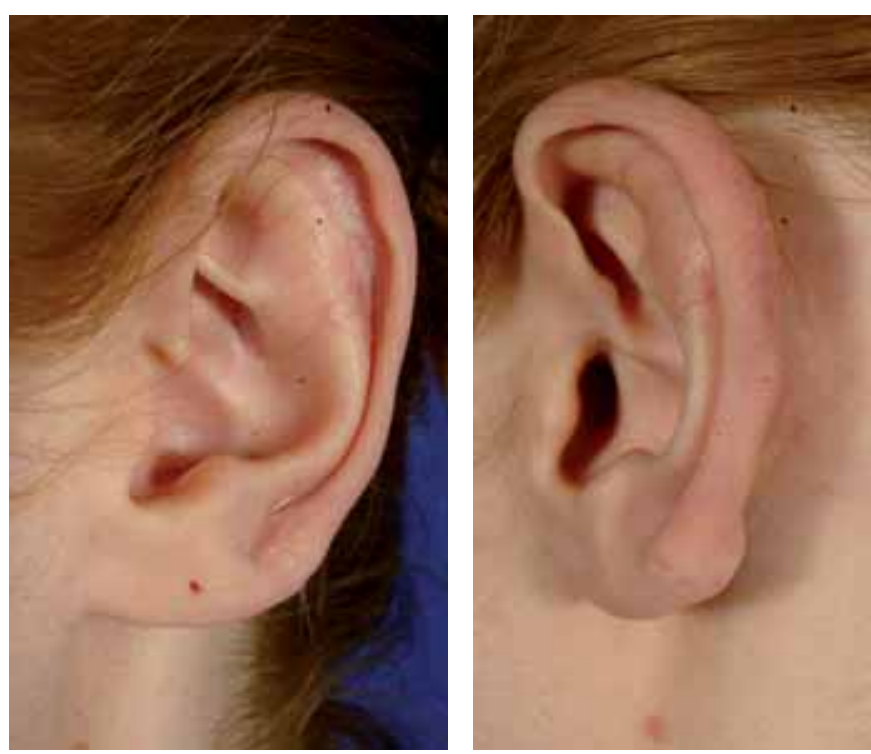

Fig. 11 y 12. Resultado tras 8 meses de tratamiento (Radioterapia externa).

blecer diferencias entre la utilización de braquiterapia o de radioterapia externa; pensamos que la causa de estas recidivas radica en la dificultad de cicatri- 


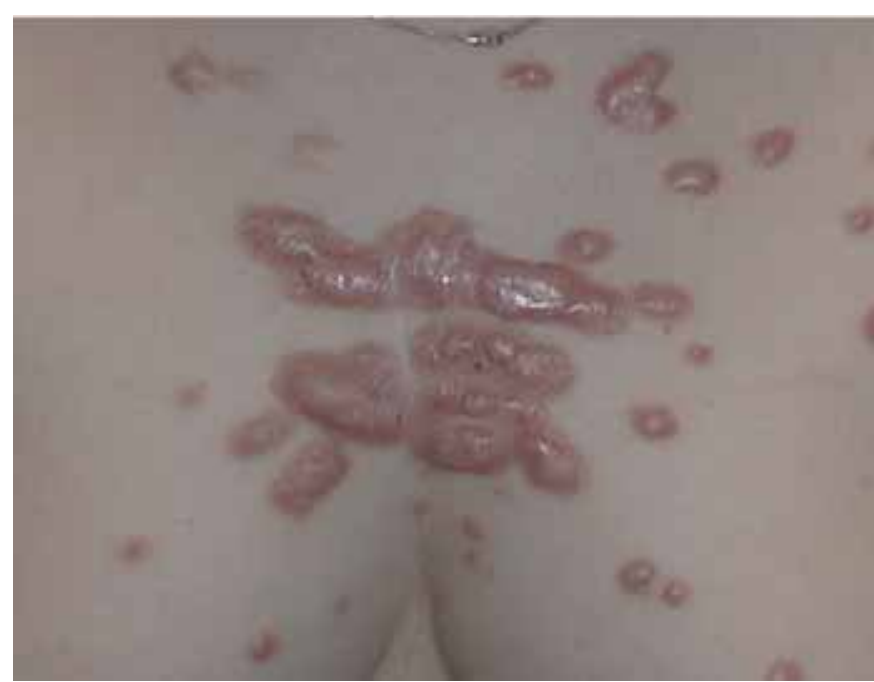

Fig. 13. Queloides múltiples en región esternal.

zación de esta zona, por pequeñas dehiscencias de sutura o retardo en el tiempo de curación. Las recidivas parciales, entendiendo como tales aquellos queloides que han mejorado mucho pero que dejan como resultado el equivalente a una cicatriz hipertrófica, están alrededor del 14\%. Las sensaciones de prurito, quemazón o hipersensibilidad desaparecieron en el $100 \%$ de los casos. Como efectos secundarios observamos telangiectasias en el 15,4\% o cambios en la pigmentación cutánea en el 5,9\%. Es frecuente a los pocos días de efectuado el tratamiento que se aprecie una discreta hiperpigmentación en la zona tratada que se resuelve sin mas tratamiento en las siguientes 6 a 12 semanas (Fig. 3-16).

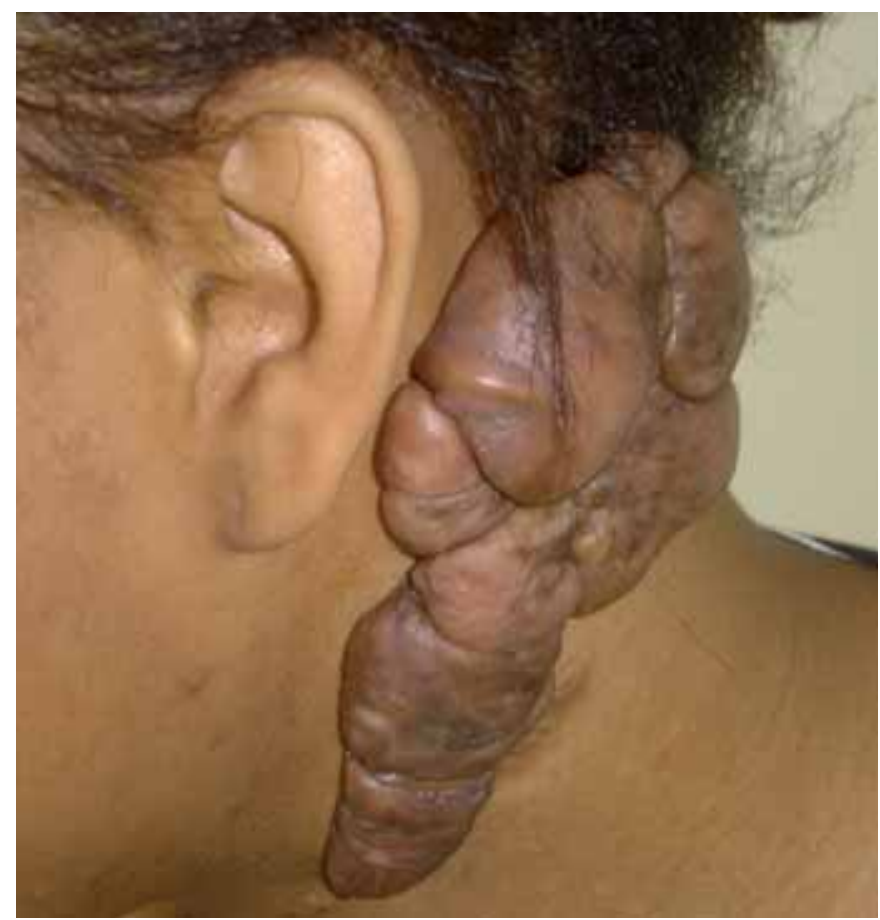

Fig. 15. Queloide de grandes dimensiones en la región retroauricular y submandibular.

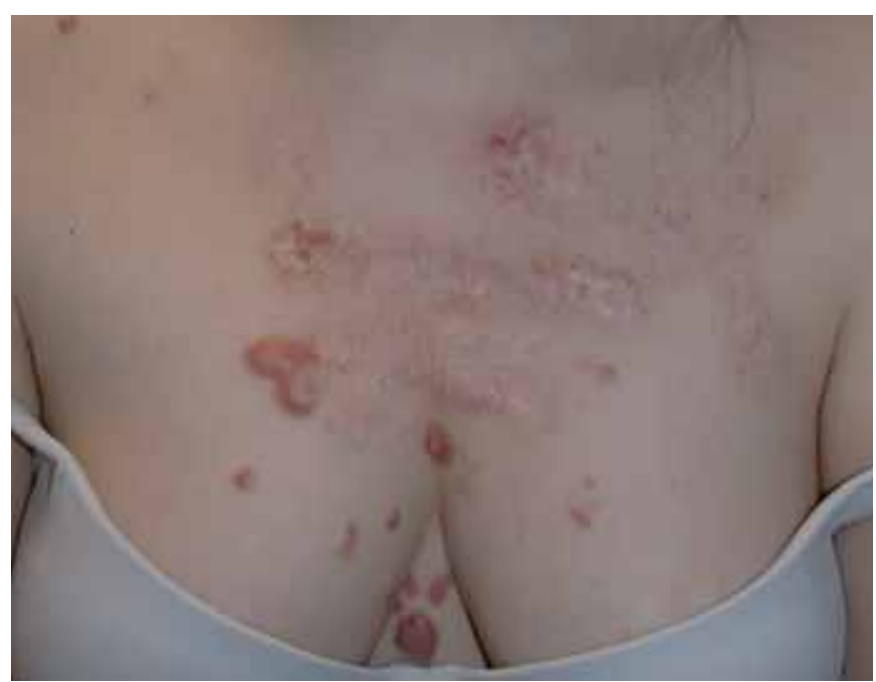

Fig. 14. Resultado tras un tratamiento en dos etapas (Radioterapia externa).

\section{Discusión}

La conveniencia de la radioterapia como tratamiento complementario a la cirugía de los queloides fue ampliamente demostrada por Borok y colaboradores (12). Ollstein (17) describió unas recidivas del $21 \%$ en 68 queloides tratados mediante resección quirúrgica seguida de radioterapia. Kovalic (18) encontró un $27 \%$ de recidivas tras un periodo mínimo de seguimiento de 2 años en 113 queloides tratados mediante cirugía y radioterapia. Bertiere (11) empleó la cirugía seguida de Iridio de baja tasa de dosis y describió un $13 \%$ de recidivas en 46 queloides seguidos durante 10 meses. Clavere (13) encontró un $12 \%$ de recidivas

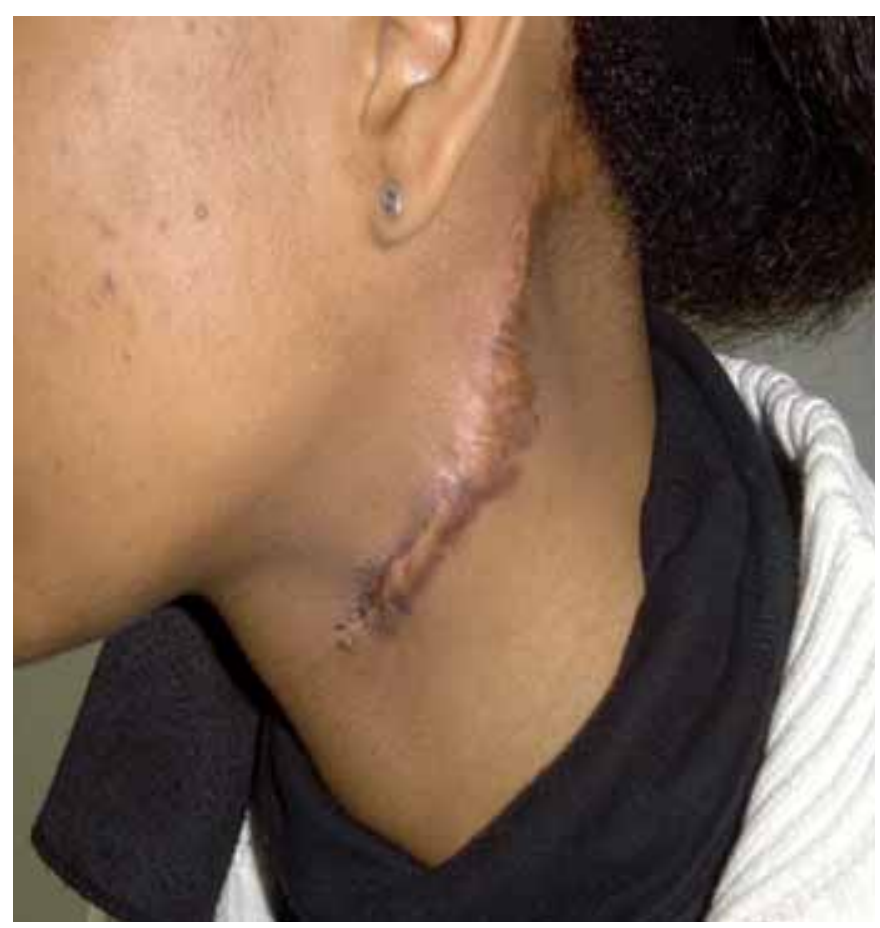

Fig. 16. Resultado al año de tratamiento (Braquiterapia). 
en 27 queloides tratados mediante cirugía e implante de Iridio 192 de baja tasa de dosis. El trabajo que incluyó más pacientes fue el de Escarmant (19), quien trató 855 queloides con radioterapia intersticial inmediatamente después de la escisión quirúrgica, encontrando una tasa de recidivas del $21 \%$. Todos estos trabajos ofrecieron cifras de control local muy superiores a las publicadas con las otras técnicas, por lo que la resección quirúrgica seguida de irradiación es considerada como el tratamiento de elección de los queloides (20).

Ninguno de los trabajos mencionados reveló diferencias en el control local ni en las complicaciones entre los casos tratados con radioterapia externa y braquiterapia intersticial de baja tasa de dosis, siendo los resultados de ambas técnicas muy comparables. El elegir entre una $u$ otra (entre radioterapia externa y braquiterapia) vendrá determinado por su disponibilidad, la situación anatómica del queloide, su forma, tamaño y por el confort del paciente.

En la mayoría de los casos de nuestro estudio el tratamiento se realizó de forma ambulatoria. El mayor inconveniente fue que el paciente debía acudir a realizar 4 sesiones de tratamiento en un corto periodo de tiempo ( 24 a 36 horas), por lo que se tenía de desplazar hasta el centro médico.

La braquiterapia de alta tasa de dosis fue un tratamiento muy bien tolerado. Ninguno de los 48 pacientes tratados se quejó de dolor o experimentó sensación de disconfort durante el tiempo que llevó el catéter. No se tuvo que retirar la aplicación antes de tiempo en ningún caso.

Ningún paciente presentó efectos secundarios grado 3 ó 4 . En los casos más desfavorables se observó la aparición local de telangiectasias, que seguramente estuvieron en relación con zonas de piel que quedaron más próximas al catéter y que por tanto recibieron dosis ligeramente más altas.

La braquiterapia de alta tasa de dosis permitió realizar una dosimetría muy precisa, y optimizar la distribución de dosis, evitando la existencia de áreas frías o calientes a lo largo del volumen del implante, de ahí la baja tasa de recidivas y los excelentes resultados estéticos conseguidos. Para ello es necesario llevar a cabo un riguroso programa de garantía de calidad (21), lo que requiere gran meticulosidad a la hora de realizar toda la aplicación y se refleja sin duda, en una mejoría de los resultados.

La contribución del cirujano plástico a la hora de obtener los mejores resultados estéticos es evidente. De su habilidad en traumatizar mínimamente los tejidos sanos vecinos y de su capacidad para evitar las tensiones cutáneas innecesarias, dependerá en gran medida que la cicatriz resultante quede con el mejor aspecto posible. Así mismo será imprescindible que la reconstrucción de los defectos provocados por el queloide se haga en un tiempo, antes de la radioterapia.

\section{Conclusiones}

Pensamos que el tratamiento combinado de exéresis quirúrgica de la cicatriz queloidea y radioterapia posterior inmediata, es un método efectivo y de elección para el tratamiento de los queloides. Es un tratamiento indoloro, no requiere ingreso, no tiene contraindicaciones, puede hacerse bajo un elevado control y por consiguiente tiene un riesgo mínimo de producir secuelas.

Finalmente resumimos lo que serian las recomendaciones básicas de este tratamiento.

1. Iniciar el tratamiento lo antes posible, a poder ser dentro de las 2 horas posteriores a la extirpación quirúrgica del queloide, para limitar al máximo la exposición de las células a los factores de crecimiento.

2. Realizar una hemostasia cuidadosa y emplear sutura subcutánea para liberar tensión, seguida de sutura intradérmica del plano más superficial.

3. Administrar radiación en dosificación 4x500cGy.

4. Realizar una optimización geométrica de la distribución de la dosis.

5. Limitar la dosis en los extremos de la lesión.

6. Administrar siempre antibióticos profilácticos.

7. En determinadas regiones como por ejemplo el lóbulo de la oreja, proteger las zonas vecinas de riesgo, como el cuello, con lámina de plomo.

\section{Dirección del autor}

Dr. Javier Bisbal.

$\mathrm{P}^{\mathrm{o}}$. Manuel Girona, 66, $4^{\mathrm{o}} 1^{\mathrm{a}}$.

08034 Barcelona. España

e-mail: 7193jbp@comb.es

\section{Bibliografía}

1. Tredget EE, Nedelec B, Scott PG, Ghahary A.: "Hypertrophic scars, keloids, and contractures; the cellular and molecular basis for therapy". Surg Clin North Am. 1997; 77:701.

2. Apfelberg DB, Maser MR, White DN, Lash H: "Failure of carbon dioxide laser excision of keloids". Laser Surg. Med; 1998: 388.

3. Cosman. B, Crikelair. GF, Ju. DMC, Gaulin. JC, Lattes. R.: "The Surgical treatment of keloids". Plas. Reconstr. Surg. 1961; 335 .

4. Kiil J.: "Keloids treated with topical injections of triamcinolone acetonide". Scand. J. Plast. Reconstr. Surg. 1977: 169.

5. Zouboulis CC. Blume U, Buttner P. et all: "Outcomes of cryosurgery in keloids and hypertrophic scars: a prospective consecutive trial of case series". Arch Dermatol. 1993; 129:1146.

6. Sherman R, Rosenfeld H.: "Experience with the Nd YAG laser in the treatment of keloid scars". Ann. Plast. Surg. 1988: 231. 
7. Ahn St, Monafo WW, Mustoe TA.: "Topical silicone gel for the prevention and treatment of hypertophic scar". Arch. Surg. 1991; 126: 499.

8. Mercer, NS: "Silicone gel in the treatment of keloid scars". Br.J.Plast.Surg. 1989: 83,

9. Ohmori, RN.: "Effectiveness of silastic sheet coverage in the treatment of scar keloid (hypertrophic scar)". Aesth. Plast.Surg. 1988: 95.

10. Wittenberg GP, Fabian BG, Bogomilsky JL. et al.: "Prospective, Single-blind, Randomized, Controlled Study to Assess the Efficacy of the 585-nm Flashlamp-Pumped Pulsed-Dye Laser and Silicone Gel Sheeting in Hypertrophic Scar treatment”. Arch Dermatol. 1999; 135:1049

11. Bertiere. MN; Jousset C; Marin JL; et al.: "Intèrêt de l'irradiation interstitielle des cicatrices chèloides par Iridium 192". Ann. Chir. Plast. Esthet. 1990; 27.

12. Borok TL; Bray M; Sinclair I; Plafker J; Labirth L; Rollins C.: "Role of ionizing irradiation for 393 keloids". Int.J.Radiat.Oncol.Biol.Phys. 1988; 15:865.

13. Clavere P; Bonnafoux-Clavere A; Roullet B; Morzel A; Rhein B; Bonnetblanc JM; Olivier JP.: "Curithérapie postopératoire des cicatrices chéloides". Bull. Cancer Radiother. 1993; 9.

14. Guix B; Finestres F, Tello JI et al.: "Seven year experience of hdr brachytherapy as treatment of keloids". Radiother. Oncol. 1999; 40(supp 1): 10-11
15. Guix B, Henriquez I, Andres A, Finestres F, Tello JI, Martinez A.: "Treatment of keloids by high-dose-rate brachytherapy: A seven-year study". Int J Radiant Oncol Biol Phys. 2001; 50:16.

16. Chang HS, Hom DB, Agarwal RP, Pernell K, Manivel JC, Song C.: "Effects of basic fibroblast growth factor on irradiated porcine skin flaps". Arch. Otolaryngol. Head Neck Surg. 1998; 124:307.

17. Ollstein, RN; Sieguel, HW; Gillooley, J; Barsa, JM: "Treatment of keloids by combined surgical excisión and inmediate post-operative X ray therapy". Ann. Plast. Surg. 1981;282.

18. Kovalic, JJ; Perez, CA: "Radiation therapy following keloidectomy: A 20 year experience". Int. J.Radiat. Oncol. Biol. Phys. 1989: 77.

19. Escarmant, P; Zimmermann, S; Amar, A; Raroanna, JL; Moris, A; Azaloux, H; Francois, H; Gosserez, O; Michel, M; G'Baguidi, R: "The treatment of 783 keloid scars by iridium 192 interstitial irradiation after surgical excision”. Int. J. Radiat. Oncol. Biol. Phys. $1993 ; 245$.

20. Guix, B; Henriquez, I; Finestres, $\mathbf{F}$ et al: "Treatment of keloids by high-dose-rate brachytherapy". Int. J. Radiat. Oncol. Biol. Phys, 2001; 50:167.

21. Guix, B; Tello, JI.: "Control de calidad de la dosimetría de los equipos de braquiterapia con fuentes de Iridio 192 de alta tasa de dosis". Oncología, 1995, 18:561. 